\title{
Correction to: When Prevention Makes Good Music
}

\author{
Carina Joly
}

\section{Correction to: Chapter 20 in: S.-H. Lee et al. (eds.), Perspectives in Performing Arts Medicine Practice, https://doi.org/10.1007/978-3-030-37480-8_20}

The original version of this chapter was inadvertently published with an error in the figure citation, Fig. 20.3b. This has now been corrected as shown below:

In a standing position, the pelvis also plays an important role as it directly affects the upper body alignment. While standing up, through the practice of a sequence of alternating anterior and posterior pelvic tilts, participants are able to identify their pelvic postural habit. Due to long hours of activities in seated position, the iliopsoas muscle group may be shortened in certain individuals who have the tendency to stand throwing the weight toward the front (Fig. 20.3a). In these cases, iliopsoas stretches are highly recommended in order to enrich the amplitude of the pelvic movements and to bring more comfort when these individuals seek the neutral pelvic position. 